\title{
NEW METHODOLOGIES FOR THE DOCUMENTATION OF FORTIFIED ARCHITECTURE IN THE STATE OF RUINS
}

\author{
F. Fallavollita ${ }^{\text {a }}$ A. Ugolini ${ }^{\text {a }}$ \\ ${ }^{\text {a }}$ Dept. of Architecture, University of Bologna, Viale del Risorgimento, 2, 40136 Bologna, Italy - (federico.fallavollita, \\ andrea.ugolini)@unibo.it
}

KEY WORDS: Castles, Laser Scanner, Digital Photogrammetry, Architectural Survey, Restoration

\begin{abstract}
:
Fortresses and castles are important symbols of social and cultural identity providing tangible evidence of cultural unity in Europe. They are items for which it is always difficult to outline a credible prospect of reuse, their old raison d'être- namely the military, political and economic purposes for which they were built- having been lost. In recent years a Research Unit of the University of Bologna composed of architects from different disciplines has conducted a series of studies on fortified heritage in the Emilia Romagna region (and not only) often characterized by buildings in ruins. The purpose of this study is mainly to document a legacy, which has already been studied in depth by historians, and previously lacked reliable architectural surveys for the definition of a credible as well as sustainable conservation project. Our contribution will focus on different techniques and methods used for the survey of these architectures, the characteristics of which- in the past- have made an effective survey of these buildings difficult, if not impossible. The survey of a ruin requires, much more than the evaluation of an intact building, reading skills and an interpretation of architectural spaces to better manage the stages of documentation and data processing. Through a series of case studies of fortified buildings in ruins, we intend to describe the reasons that guided the choice of the methods and tools used and to highlight the potentials and the limits of these choices in financial terms.
\end{abstract}

\section{INTRODUCTION}

\subsection{Introduction}

Fortified buildings are assets scattered around Italy and Europe. Their construction has always changed the landscape, marking the tracks of populations, describing the methods of supervision and occupation of a territory, fulfilling a strategic bulwark to protect an inhabited area and providing a valuable refuge for rural populations in case of danger.

In recent years, a research unit from the University of Bologna composed of architects from different disciplines has conducted a series of studies on the fortified heritage of Emilia Romagna often characterized by buildings in ruins. It is a series of studies the purpose of which is to document assets investigated in depth by historians but which, however, lacked reliable surveys in order to define a credible as well as sustainable conservation project. In the prologue of the De re aedificatoria, Leon Battista Alberti reminds us that «...nel vedere l'opera d'altri architetti subito percorriamo con lo sguardo e valutiamo una ad una le misure dell'edificio» ${ }^{1}$. It is through measurements that we try to reproduce space and objects adapting the complexity of reality to models that are supposedly reliable and can be interpreted from many points of view. Models to be understood not as the culmination of the measurement procedure, but as an intermediate-so to speak "provisional"- processing stage from which to carry out further intensive developments ${ }^{2}$.

Technologies and digital models have been put side by side with traditional survey techniques and are changing modalities, acquisition timelines and methods for processing data. These systems, in constant and rapid evolution, permit you to acquire a lot of information about an architectural object, but we are convinced that they require the technical mastery and critical

1 Alberti 1966, p.10.

2 Torsello 1988, pp.131-139. skills of a "connoisseur of architecture"3 on the part of those who use them (FF, AU).

\section{FORTIFIED ARCHITECTURES IN RUINS}

\subsection{Fortified Architectures in Ruins}

A great deal has been written about the fact that fortresses and castles are important symbols of social and cultural identity as tangible symbolic evidence of cultural unity in Europe. Mostly abandoned, today these architectures appear difficult to study because of the lack of archive documents available; difficult to approach due to the impervious nature of the sites where they are located and the close relationship they have interwoven with the nature that surrounds them (and which often invades them); difficult to investigate because of their size and articulation, which is often the result of successive layers and modifications in their use; but especially difficult to analyse because of their state of preservation being, in most cases, that of ruins, the latter making them ideal for the study of techniques and building materials in the field of material culture.

A building, as is known, always bears witness to itself as through its materiality and signs it is able to tell its own story. Its understanding requires at least basic knowledge of what the principles that governed the building of the castle were, in order to search for and/or recognize the signs that characterize this type of architecture. Recognition not only in terms of a comparison of stories and images, but also knowledge of the criteria that governed the building of a castle, the construction techniques and materials used.

A castle, after all, consisted of a few elements and by recomposing them it is possible to obtain the infinite variety of all European castles. We can find castles made up of only a main tower, the palatium, the fortified manor house, the walls, the 


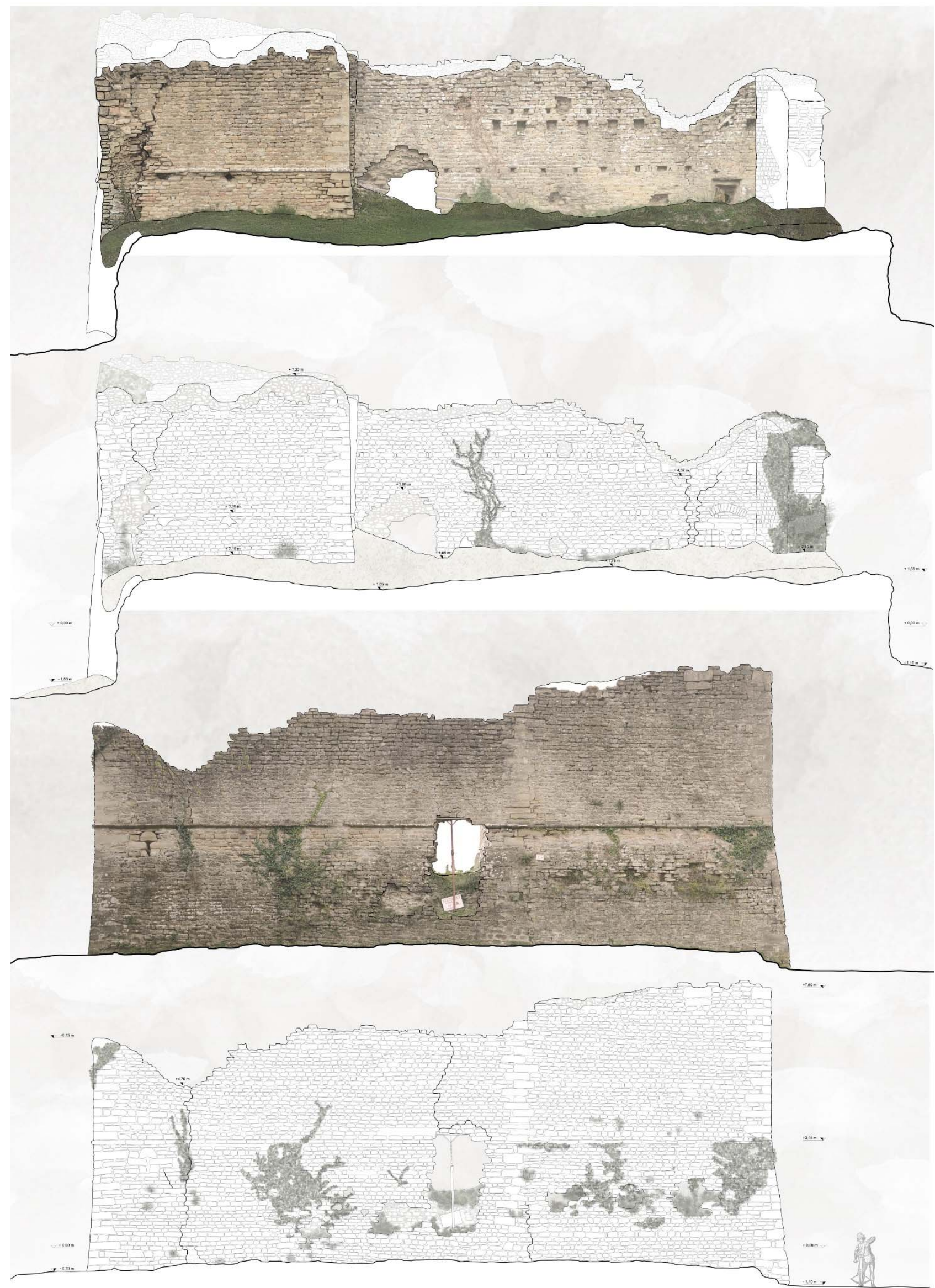

Figure 1. Cantagallo, photogrammetry and elevations NE (surveyors M. Morra, S. Ferraiolo) 

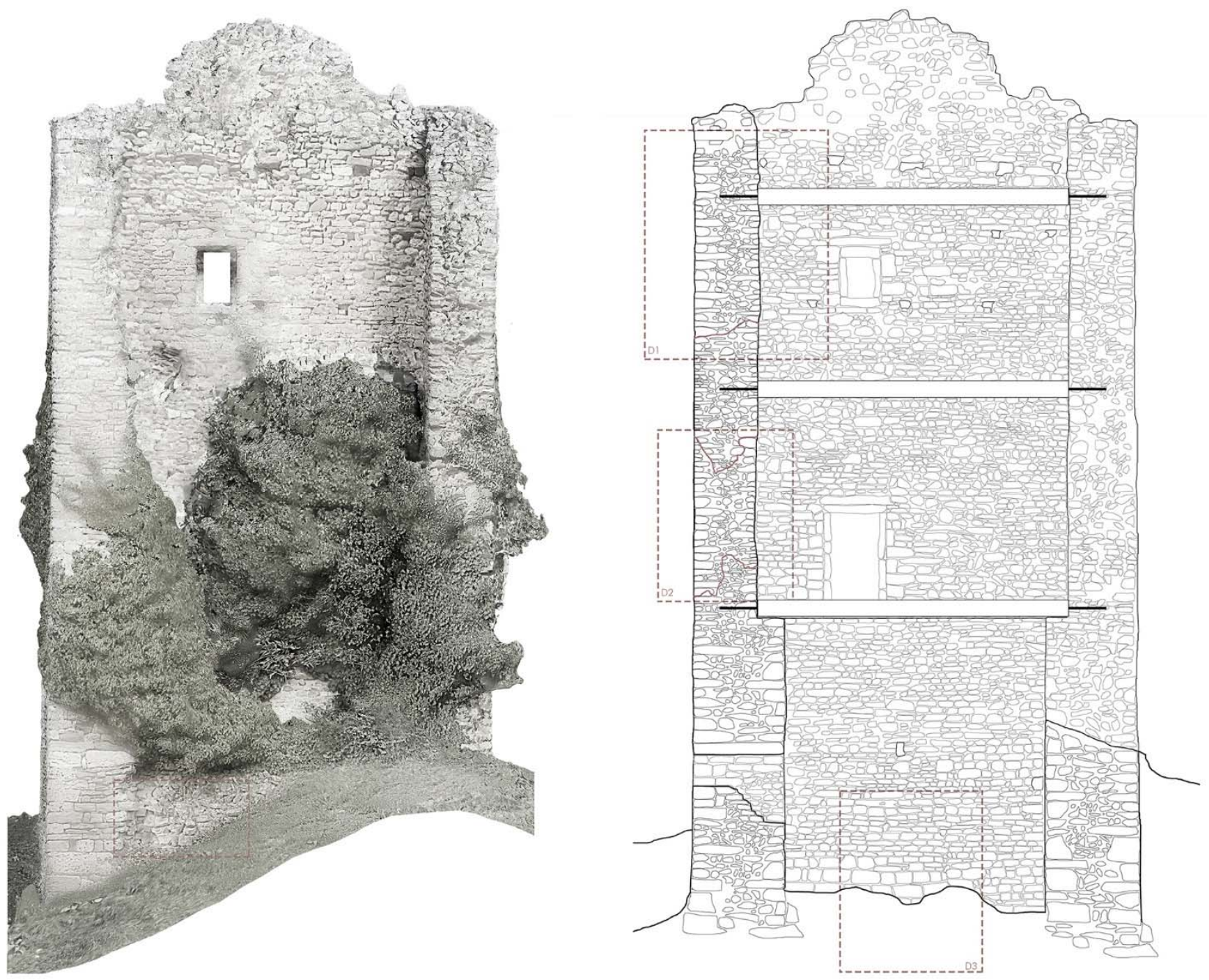

Figure 2. Castelnuovo, section of the main tower (surveyor M.Sansavini)

actual defensive perimeter, sometimes marked by towers or a mixture of all these elements, in addition varying in number. These are the constituent elements of the fortified organism that some scholars call primary macro elements to which defensive complements are necessarily related, such as: the merlon, the wall walk, the brattice, the scarp and counter-scarp, the slits, the barbican and the outer wall which contribute together with the type of organization of the buildings and the construction techniques used, to the dating of the artefact ${ }^{4}$.

Locating and documenting the exact design and articulation of a castle and its components, especially if the artefact is in ruins, in recent years has enabled us to better understand the ratio and phases of construction and transformation of this kind of building. The choice of their plan, for example, was often dictated by the location in which the edification of the fortress was determined: in the mountains irregular shapes were the rule, as was imposed by the need to adapt to the morphology of the site. In lowland locations, however, the influences may derive from existing walls or, in cities, by considerations on land property.

Studies in recent years have involved forts placed on hills or mountains, on plains and urban areas. They are examples of buildings dating back to between the end of the tenth century and

4 Hislop 2014; Palloni.

5 The first to adopt this definition was Carlo Perogalli in G.C. Bascapè, C. Perogalli, Torri e castelli di Valtellina $e$ the fifteenth century, a period in which the updating of types of medieval defense and reinforcement of pre-existing artefacts was witnessed. The research has included various types of fortifications from single castles such as Pierle, to the enclosed castles $^{5}$ such as Castrum Cantagalli, near Imola or Castrum novi, near Forli, to fortified defense areas in towns now partially or completely disappeared such as Castrum Corzani in San Piero in Bagno or Castrum Pianetti near Meldola, Castrum Scortigate or Villa Corliani (turned into a castle in the late fifteenth century), Tumba Gathei, then castrum, near Rimini, up to more complex and articulated fortresses such as Castrum Mons belli of Modigliana, Castrum Carium today Castrocaro, the castle of Ravaldino or the Sforzesca in Forlì (AU).

\subsection{Observation and Measurement}

Simple direct observation, although essential for this kind of artefacts is often not enough to decipher the complexity of visible signs that characterize them and testify their history, and requires the use of analytical scientifically-based techniques. The human eye is unable to grasp exactly the metric properties of things and the measure becomes the tool that makes it possible to describe the quality of the phenomena and their effects. That is why we

Valchiavenna, Edizioni Banca Piccolo Credito Valtellinese, Sondrio, 1966. 

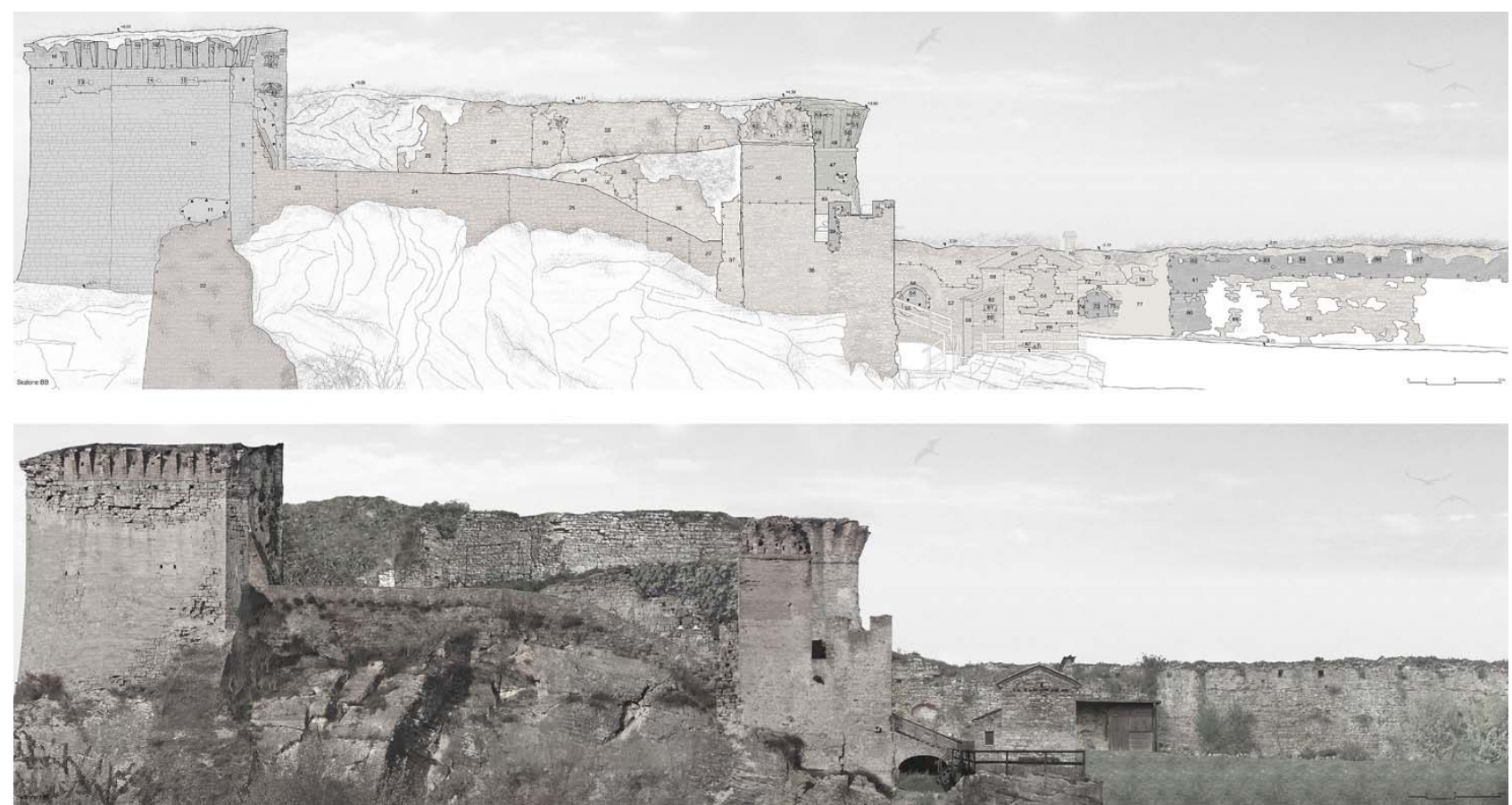

Figure 3. Castrocaro, photogrammetry and sections of S side (surveyors S. Iosca, E. Fantini)

can say that a survey is an analytical practice having as its subject the geometry of built architecture. Then we can talk about "intentional" geometries that are those which make reference to the architects and "accidental" geometries that tell about mistakes, executive abnormalities, instability and the degradation of a building ${ }^{6}$.

In these years through the geometry of buildings we have been able to retrace the skills of those who built these castles, the techniques in use in those places, site movements and the arrangement of structures, in brief, many of the events that have marked their life. In Cantagallo, for example, a series of large blocks, similar to those used in the corners, along the boundary marks the presence of the main tower by reinforcing the wall. A mistake in the bedding of the radial buttresses of the Modigliana fortress, the origin of the partial collapse of the imposing main tower allowed us to document how they created it. The beam hole visible on the vestments of Castelnuovo or Pianetto allowed for the tracing of the position of missing wooden slabs and the understanding of what type of scaffolding was used for the edification of the tower (fig. 1). The reading of the high stratigraphy, thanks to the high precision of the processed drawings, provides a valuable aid to the study of the processing stages of these artefacts and the detection, as in Corzano, of recent restorations. The geometry of a wall, changes in the materials, and the marks left by missing decks or construction gimmicks like a drawbridge allowed for the tracing of the uses and the history of a castle. Observing these signs and geometric anomalies under another point of view, in many cases, made it possible to identify and interpret disturbances underway and forms of degradation and report expected vulnerabilities; which are no marginal aspects for those involved in building conservation. A series of scaffolding holes or the change of wall thickness due to the absence of a floor, for example, may turn into "hinges" that favour the overturning of a portion of the wall as can be seen in Castelnuovo (fig. 2). The presence and progress of lesions in corners facing NO and SE of the Cantagallo mastio indicate the dangerous expulsion of the corner building. The outward deformation from the plane of a wall septum allowed for the evaluating of the severity of its rollover while the geometry of collapses such as those in the ruins of the fortress Pierle allows the reading of a parabolic collapse due to horizontal forces such as those of an earthquake (fig. 3). Accuracy of measurement becomes the par excellence means to identify diversity, and state that what appears as uniform is actually manifold (fig. 4) (FF, $\mathrm{AU})$.

\subsection{Potentials and Critical Issues in New Survey Methods}

In the last fifteen years, digital technologies have revolutionized survey standard methods: today the scientific architectural survey is done mainly with the help of laser scanning and digital photogrammetry. Compared to traditional methods, direct and instrumental, times and approach modes to the artefact to be evaluated have changed. Castle ruins are a paradigmatic example for highlighting the strengths and weaknesses of these changes.

The stages of an architectural survey can be basically divided into two main parts: a first stage of data acquisition and a second stage of selection, interpretation and restitution of data. The first step of course is always done "in the countryside", i.e. involves the collection of measurements of the artefact. This collection can be made directly, i.e. with metric rods and tape measuring, or with specific tools such as laser scanners. The second step, however, always takes place "in the studio" and relates to the interpretation of the data and the production of $2 \mathrm{D}$ and $3 \mathrm{D}$ models of the artefact. We can say that the first stage of the survey is purely quantitative and concerns objective data. The second, however, is also qualitative and strongly affects surveyor culture. In traditional surveys, timing and the importance of the two stages were equivalent. This was due to the fact that the first phase of data collection also consisted in the selection and interpretation of data. The preparation of a preliminary survey and the selection of points to be measured were fundamental to the second phase. The survey had to be designed and carefully planned from the first step. The survey and direct contact with the artefact were essential for the preparation of the preliminary survey. In the preliminary stage you were also required to know how to 


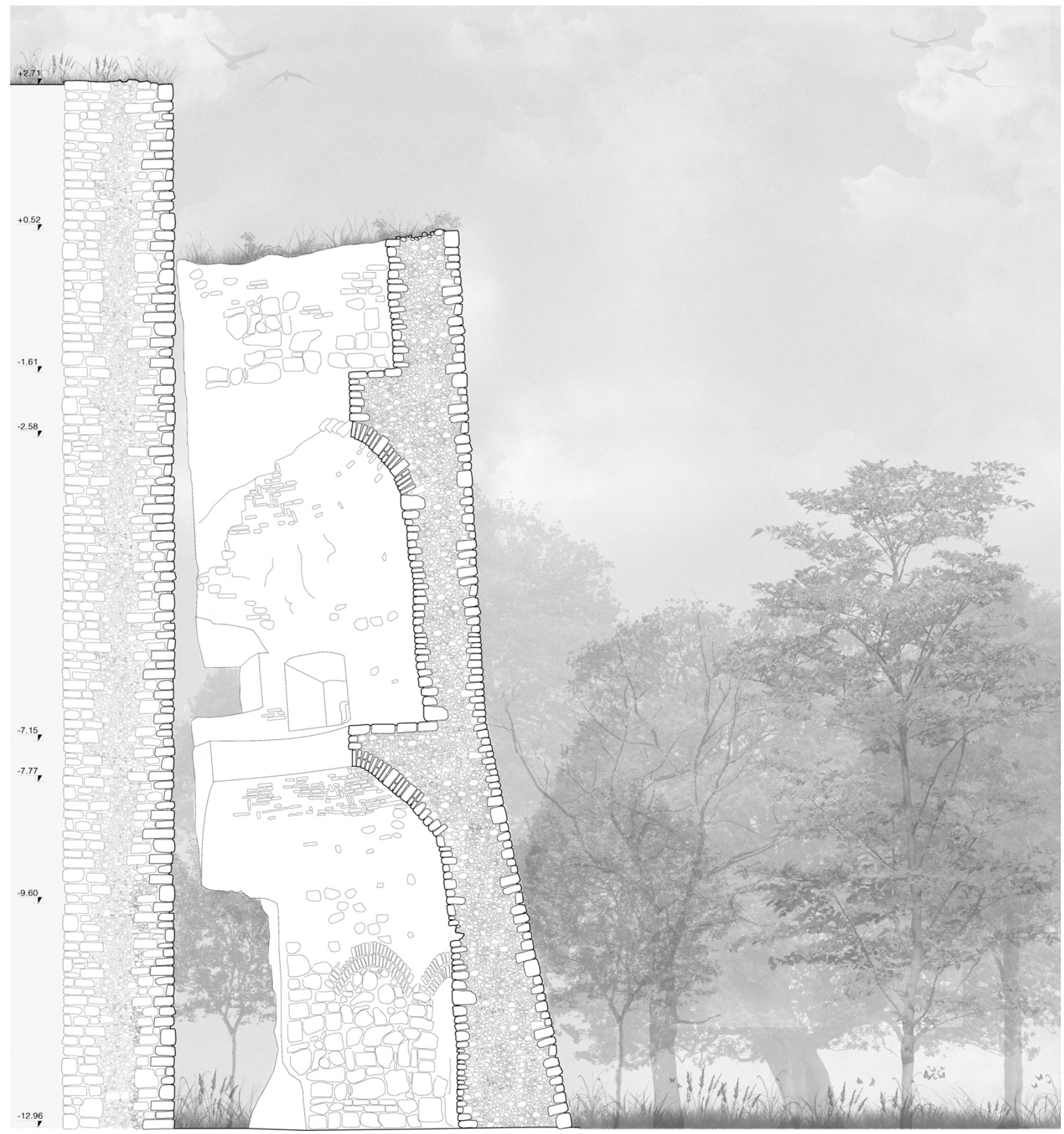

Figure 4. Castrocaro, section tower NE (surveyors S. Iosca, E. Fantini)

interpret the data beforehand: that is, you were supposed to predict what plans, sections and elevations needed to be drawn. With the new digital technology, times and the importance of the two phases have changed. The first phase of data collection compared to the total time devoted to the survey covers only a small part: it can basically cover the time needed for the positioning of the laser scanner or the photo shoots. Prolonged direct contact with the artefact is no longer necessary to produce an accurate preliminary survey and take measurements. The selection and interpretation of data is entirely confined to the second phase, to be carried out in the studio. Today this last stage has become crucial and relates mainly to the surveyor's culture. This change has both positive and negative aspects. On the positive side there is first of all the accuracy and communicability of the data collected. It is a known fact that a reliable scientific survey must start from data collection being as objective and repeatable as possible. In this respect, the contribution of digital tools is remarkable. The ability to store large amounts of data in a short time is an undeniable advantage. Furthermore, data has became more reliable than traditional measures. This is because the gap is often reduced by the tolerance of the instruments used; tolerance now in the order of a few millimetres.

Reducing time to campaign also leads to greater security for the surveyor. In fact, some sites analysed are dangerous because their conservation status is often precarious. In some cases we have decided to use a drone to survey the entire site because any other option was not feasible given the topography of the surrounding terrain.

The accuracy of the data detected, given the predictable and calculable errors, allows you to interpret and determine with a 


\begin{tabular}{|c|c|c|c|}
\hline year & object & the survey authors & equipment \\
\hline \multirow[t]{2}{*}{2011} & Rocca di Montebello & C. Mariotti, S. Mencarelli & Total Station + Photoplan \\
\hline & Castello di Zocco & S. Fabbretti & Total Station + Photoplan \\
\hline \multirow[t]{2}{*}{2013} & Rocca di Pierle & F. Ranicchi, B. Braho & Total Station + Laser Scanner \\
\hline & Rocca di Forlì & R. Novelli, C. Castellari, L. Salvetti & Total Station + Photoplan \\
\hline 2014 & Rocca di Pianetto & M. Amici, E. Ceccaroni, L. Salina & Total Station + Photoplan \\
\hline \multirow[t]{2}{*}{2015} & Castello di Coriano & F. Galli, C. Magnani, D. Zenoni & Total Station + Photogrammetry \\
\hline & Rocca di Modigliana & F. Bedeschi, E. Festa; G. Castellari, F. Morsiani & Total Station + Laser Scanner \\
\hline 2016 & Fortezza di Castrocaro & S. Iosca, E. Fantini & $\begin{array}{l}\text { Total Station + Laser Scanner + } \\
\text { Photogrammetry }\end{array}$ \\
\hline 2016 & Castello di Cantagallo & S. Ferraiolo, M. Morra & Total Station + Photogrammetry + Drone \\
\hline 2016 & Castello di Corzano & G. Denicolò, S. Fratti & $\begin{array}{l}\text { Total Station + Laser Scanner + } \\
\text { Photogrammetry + Drone }\end{array}$ \\
\hline 2016 & Rocca di Castelnuovo & M.Sansavini & $\begin{array}{l}\text { Total Station + Laser Scanner + } \\
\text { Photogrammetry + Drone }\end{array}$ \\
\hline 2016 & Castello di Scorticata & M.Bostrenghi, F.Grilli & Total Station + Photogrammetry + Drone \\
\hline
\end{tabular}

Table 1. Chronological list of the architectural surveys with the instruments used

high margin of accuracy the geometry of the artefact. The cloud (or mesh) of points obtained from the shooting of a laser scanner or photogrammetric allows to have a numerical model of the object to be analysed. The drawing scale of the object model is linked to the precision with which the images were taken. You can dissect and analyse the numerical model at will: at any time, you can decide to pull out the data to create another section or plan. These indisputable advantages, however, involve greater attention and culture on the part of the surveyor in the selection phase, that of the interpretation and restitution of the data. The detector is often required to possess specific knowledge of the software adopted by different systems. Software dedicated to these technologies improve every year and require constant attention: in other words, updating for surveyors is nowadays a rising need. If in the past, excellent knowledge of the methods of representation and lengthy experience in the field ensured full control of the survey, that knowledge today no longer seems to be sufficient: good architectural culture must support good technical knowledge of the digital tools used (fig. 5).

The other downside is the prolongation of the time required for data analysis; data that is often redundant and takes up a lot of virtual space. In this context, we cannot help but mention the problem of archiving digital data: who can make sure that in ten or twenty years we will be able to access the data collected? A traditional survey produced printed materials and numerical tables difficult to transmit but ensured, if well preserved, the possibility of returning to the collected data even after many years. The new digital data no longer guarantees this possibility and the scientific community of architects does not seem to understand the extent of the problem. In addition, the amount of data that a laser scanner or photogrammetry produces is usually in excess with respect to real needs.

Compared to the two adopted digital technologies, namely the laser scanner and digital photogrammetry, we noticed that digital photogrammetry in the last four years has become increasingly

\section{Gaiani 2015.}

8 The tools used in the Bologna Architecture Department are: a laser scanner Leica C10; a total station Topcon GPT-3005N; a popular (table 1). The reasons are basically two. The first concerns the use of low cost technology: to perform a good photogrammetric survey today a good commercial camera and a software are enough to manage the data and match photos ${ }^{7}$. A cheap laser scanner is in any case far more expensive than any camera. In addition, a camera can currently be mounted on a drone and allows for a flexibility of use that is superior to a laser scanner; as demonstrated by the experiments conducted in the ruins of the fortresses of Cantagallo, Castelnuovo and Corzano. The second reason is the rapid development that this technology is experiencing. Only some years ago carrying out a reliable scientific survey using digital photogrammetry by means of a commercial camera was unheard. The survey experience carried out at Pierle in 2012 only with the help of a laser scanner this year would have been tackled differently and would have also involved the use of digital photogrammetry (fig. 6). Today we can conduct entire survey campaigns through a camera as was done at the Coriano castle, where the entire perimeter of brick walls was detected by means of a commercial camera (fig. 7). However, it is always necessary to get a feedback on the correctness of the survey with other more reliable metric tools: it is advisable to compare the numerical models obtained through the photogrammetric survey with measures extracted from a total station or, better still, from a laser scanner; as was done in many of the case studies mentioned ${ }^{8}$. In the near future we will probably take sufficiently accurate measurements using our phones as measuring instruments. Compared to a laser scanner, digital photogrammetry returns much higher quality images. In the case studies examined, where the presence of stone walls and brick was normal, the technique of photogrammetry lent itself very well to the capturing of architectural surfaces. This technique is particularly suitable when building surfaces present rich information, i.e. in computer jargon present a rich texture (FF).

Camera Nikon D40 and D3100; Cyclone software for the management of the cloud points of the Leica scanners; Agisoft PhotoScan software for the digital photogrammetry. 


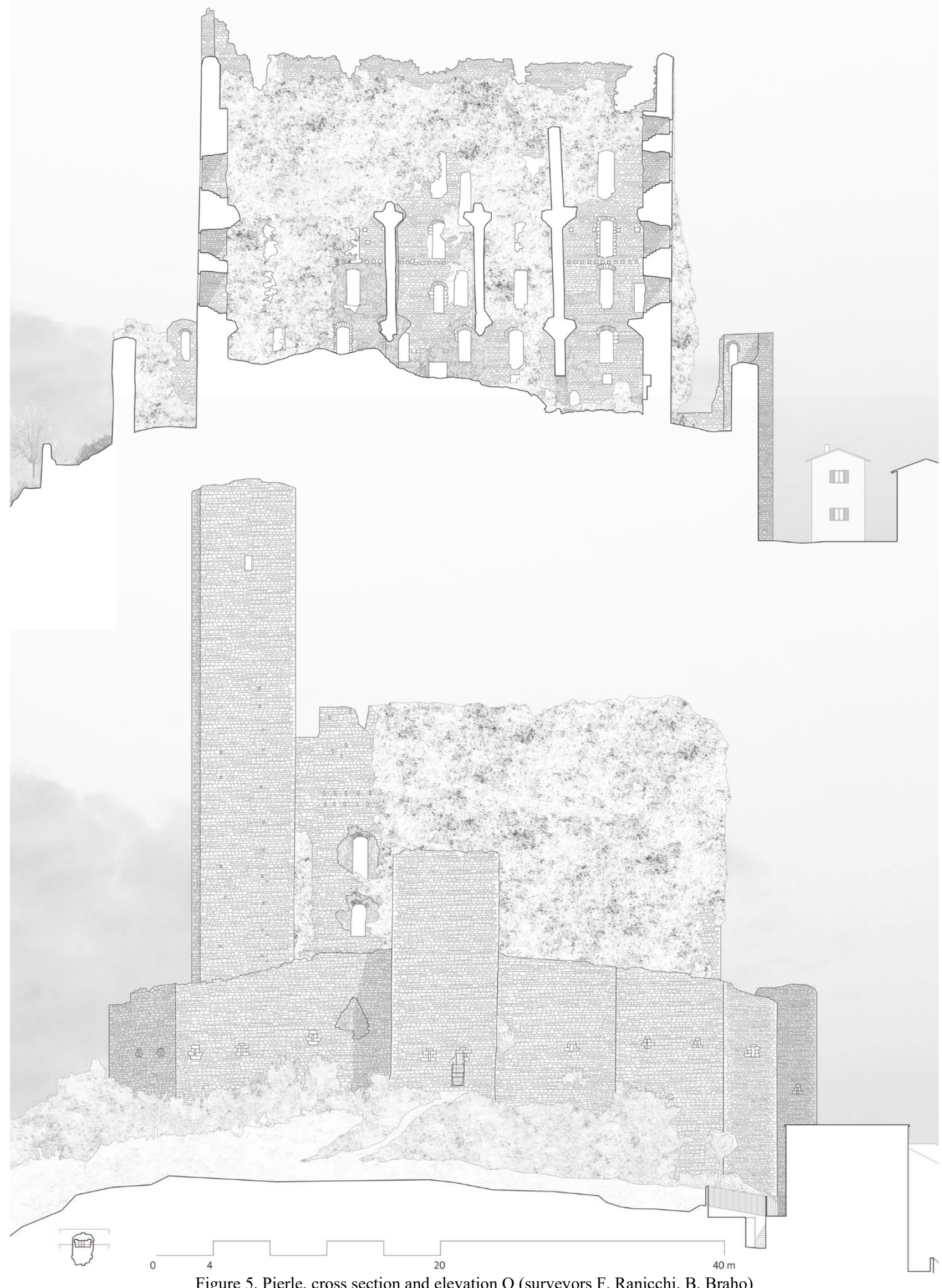

Figure 5. Pierle, cross section and elevation O (surveyors F. Ranicchi, B. Braho) 

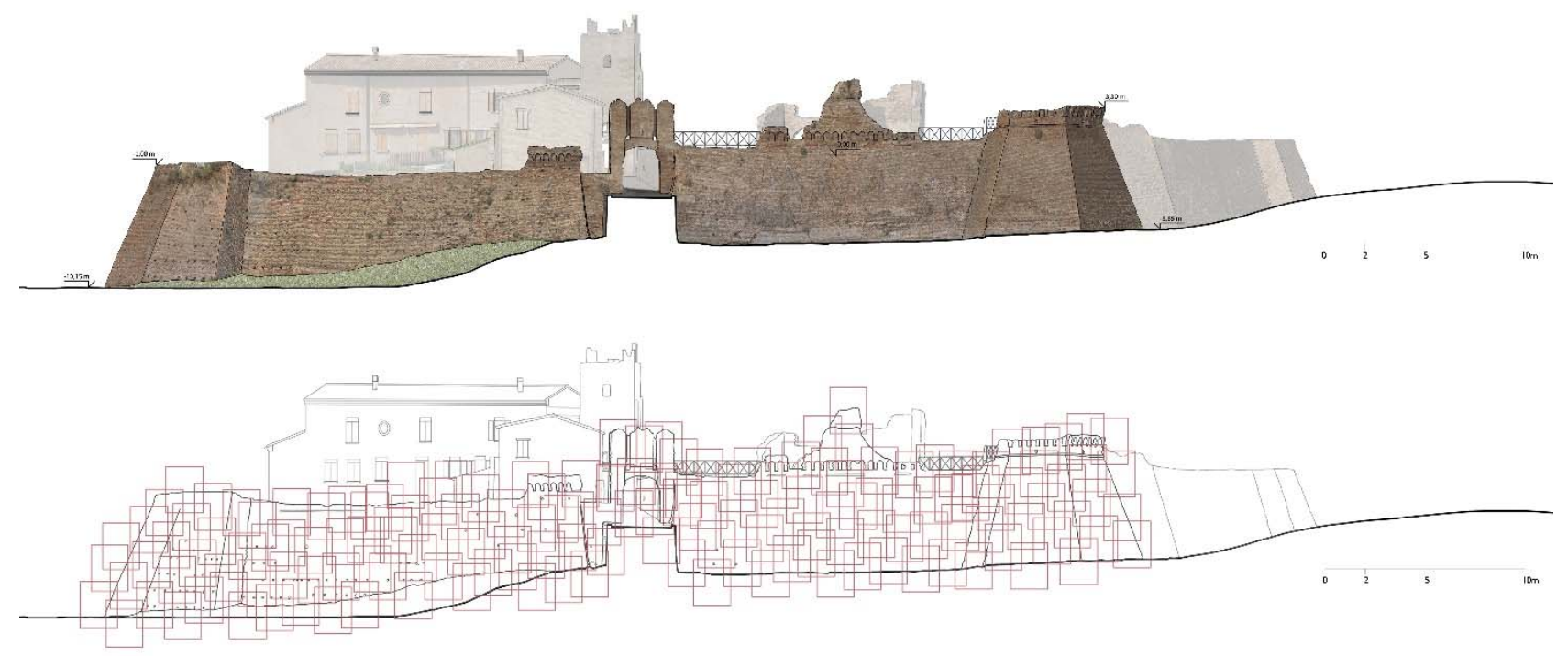

Figure 6. Coriano, photogrammetry of side S (surveyors F. Galli, C. Magnani, D. Zenoni)

\section{CONCLUSIONS}

The enthusiasm for the potentials of these digital systems has in recent years encouraged a "cloud point market", which, as is known, is not a survey but only a collection of data for the preparation of plans, prospects and sections. In addition, the inexperience of operators has led to the proliferation of this big data, the management and verification of which is quite difficult and, in some cases, this data has proven of actually little use to the project.

Aside from the problems and potentials of these digital survey methods, we believe that the surveyor's culture is still crucial to the quality of work. Despite the increasingly advanced automation seems to suggest that in a not too distant future it will be possible to obtain fully automated surveys, we think that the critical intervention of an architect will always be decisive. In addition, we believe that direct contact with the factory will help to develop "confidence" with the artefact, which is essential for the interpretation and decoding of the signs that tell us about the artefact and its space.

A great deal has been written about the fact that "in the practices of the project and in that critical survey, the cognitive process [the architectural survey] is considered a pivotal point, although not the only, (...) What directs the work of the surveyor is their ability to identify the forms that underlie the space to be detected, knowing that their task will then consist in conveying that content through words, symbols and spellings " $"$ : thus the survey is configured as a critical exercise, i.e. qualitative, prior to being quantitative. Ultimately, it should be noted that restoration aims to protect the ability to interpret the work as a source of culture ensuring the survival of data and its usability. Likewise the quality of a model and then of a survey will only be guaranteed if the outcome of the surveyor's work will configure itself as a reliable document for multiple interpretations in the future (FF, $\mathrm{AU})$.

\section{REFERENCES}

Alberti L.B., 1966. L'architettura, Il Polifilo, Milano

Bianchini C., Inglese C., Ippolito A.,2016. New Analysis About Archaeological Architecture (AA): Six Ancient Theatres of the Mediterranean Sea, in SCIRES-IT - SCIentific RESearch and Information Technology.

Gaiani M. (edited by), 2015. I portici di Bologna. Architettura, modelli $3 D$ e ricerche tecnologiche, Bononia University Press, Bologna.

Hislop M. 2013, How to Read Castles, Bloomsbury Academic.

Marconi P. 1984. Quale storia per il restauro? La figura del conoscitore di architettura moderna", in G. Spagnesi (a cura di) Esperienze di storia dell'architettura e restauro, Istituto dell'Enciclopedia Italiana, Roma.

Palloni D, http://www.icastelli.org/ (08 april 2017).

Torsello B.P., 1988. La materia del restauro, Marsilio, Venezia.

Torsello B.P., 2006. Figure di pietra. L'architettura e il restauro, Marsilio, Venezia.

Revised April 2017

9 Torsello 2006, p.32. 